\title{
Evaluation of the Effect of Gait Aids, Such as Canes, Crutches, and Walkers, on the Accuracy of Step Counters in Healthy Individuals
}

This article was published in the following Dove Press journal: Orthopedic Research and Reviews

\author{
Paul Kooner \\ Taran Schubert \\ James L Howard \\ Brent A Lanting \\ Matthew G Teeter \\ Edward M Vasarhelyi $(\mathbb{D}$ \\ Division of Orthopaedic Surgery, London \\ Health Sciences Centre, University \\ Hospital, Western University, London, \\ ON, Canada
}

Background: Wearable step-counter devices have become inexpensive tools that enable patients, researchers, and clinicians to objectively monitor physical activity. It is unknown how the use of gait aids, such as canes, crutches, and walkers, affects the accuracy of these devices. Such gait aids are commonly used by patients with chronic physical impairment and after joint-replacement surgery. The aim of this study was to determine the effect of gait aids on the accuracy of wearable step counters.

Methods: Nine healthy participants wore a Fitbit step counter on their wrist and hip and performed eight walking tests with canes, crutches, and walkers. Bland-Altman analyses were performed for all eight walking tests in order to compare agreement between measurement techniques.

Results: Mean overall agreement for subjects walking without gait aids in the hip group was excellent, showing a bias of -2.9 , with limits of agreement (LOAs) between -8.72 and 2.95. For use of canes or crutches, the Bland-Altman plots had a range of bias values between the hip and wrist counters from -7.22 to 33.56 , with LOAs from -98.55 to 124.2. The wrist counter during the four-wheeled walking test showed very little agreement with the actual step count, with a bias value of 91.33 and LOAs of 64.1-118.6, illustrating exceptionally unreliable step counts.

Conclusion: This study suggests that these widely commercially available step counters have poor reliability with gait aids, especially walkers, which should be taken into account in research and clinical settings.

Keywords: gait aids, step counters, Fitbit, monitor, physical activity, rehabilitation

\section{Introduction}

Wearable step-counter devices are increasingly being used as an objective measure of physical activity in healthy populations, with high inter- and intradevice reliability in behavioral research. ${ }^{1}$ This has provided clinicians with a valid and reliable assessment tool to measure physical activity versus historically subjective and patient-reported data. However, there is limited research on the validity of such devices in patients with physical impairments. For patients with chronic disease, such as osteoarthritis, restoring activity levels is one of the most important outcomes for clinicians. ${ }^{2,3}$ To allow patients to return to a healthy lifestyle, gait aids are commonly used during the treatment process. Therefore, objective monitoring of physical activity of patients using gait aids may be a useful tool to evaluate the effectiveness of an intervention or the rehabilitation process.
Correspondence: Paul Kooner

Division of Orthopaedic Surgery, London Health Sciences Centre, University

Hospital, Western University, 339

Windermere Road, London ON N6A

5 A5, Canada

Tel +I $519685-8500$

Email kooner.paul@gmail.com
Orthopedic Research and Reviews 2021:13 I-8

(c) $(1)(8)$ ๑) 2021 Kooner et al. This work is published and licensed by Dove Medical Press Limited. The full terms of this license are available at https://www.dovepress.com/terms. (C). work you herbby accept the Terms. Non-commercial uses of the work are permitted without any further permission from Dove Medical Press Limited, provided the work is properly attributed. For permission for commercial use of this work, please see paragraphs 4.2 and 5 of our Terms (https://www.doveperss.com/terms.php). 
Physical activity can be measured objectively through accelerometry, pedometry, heart-rate monitoring, global positioning systems, or direct observation. ${ }^{4,5}$ Historically, many of these techniques have been impractical for use outside the research setting, due to their cost and technical requirements. Patient-reported outcome measures provide subjective data and lower costs; however, they have relatively poor validity and correlation when compared to objective measures.

Wearable sensors, such as Fitbits (Fitbit, San Francisco, CA, USA), have become inexpensive activity monitors that enable patients, researchers, and clinicians to monitor numbers of steps per day. Despite the commercial availability of these monitors, few have been validated in patients with chronic impairment. Recent studies have compared the accuracy of activity-tracker devices in individuals with physical disabilities. The FuelBand (Nike, Beaverton, OR, USA), Vivofit (Garmin International, Olathe, KS, USA), and Fitbit Zip have been assessed in patients after stroke and traumatic brain injury. ${ }^{6,7}$ Overall, the Fitbit Zip was assessed to be the most accurate activity tracker for people after stroke, showing excellent accuracy and low error with relatively slower walking speeds. ${ }^{7}$ Only the StepWatch Activity Monitor (Orthocare Innovations, Oklahoma City, OK, USA) has shown higher accuracy than the Fitbit Zip in this population; however, its cost is roughly ten times that of the Fitbit Zip and it was found to be not user-friendly. ${ }^{8,9}$ Collectively, this makes the StepWatch impractical for personal use or large-scale research.

Although the use of activity trackers has been explored in patients with stroke or traumatic brain injury, it is unknown how the use of gait aids, such as walkers, canes, and crutches, affects the accuracy of these devices. Such gait aids are commonly used by patients with chronic physical impairment and after joint-replacement surgery. It is possible that the use of a gait aid may influence the measurement of steps, translating to errors in reported literature and effect-treatment outcomes.

The aim of this study was to determine the effect of gait aids on the accuracy of wearable step counters. We hypothesized that due to the change in walking mechanics with gait aids and decreased walking speeds that step counters will underestimate the step count. With this information, we may better understand the role of wearable activity monitors as a reliable, clinically applicable tool for benchmarking physical activity of individuals using gait aids.

\section{Methods}

Nine volunteers were recruited (three women, six men) for this study. Inclusion criteria included healthy volunteers with no previous orthopedic issues that were able to perform walking tests with each gait aid. Ethics were approved by the London Health Sciences Centre institutional ethics review board. This study was conducted in accordance with the Declaration of Helsinki.

Participants were asked to walk down a $20 \mathrm{~m}$ hallway twice, completing a minimum of 100 steps. An observer counted the number of steps taken using a thumb-push tally counter. Participants also wore a step counter on their waistband (Fitbit Zip hip counter) and on their dominant wrist (Fitbit Alta wrist counter). The tracker communicated with the Fitbit smartphone application on a handheld device. Standard gait aids - a cane, pair of crutches, stationary walker, and four-wheeled walker were used in this experiment. Each participant completed eight walking tests (Table 1).

The primary outcome of this paper was to assess and compare the accuracy of Fitbit step counters between the actual number of steps, steps counted by the waist-worn sensor, and steps counted by the wrist-worn sensor. Comparisons were made using ANOVA for each test with $80 \%$ power to detect differences of more than one step between measurement methods, assuming $\alpha=0.05$ and error of 1 . The Tukey post hoc test was used to compare measurements between the waist-worn and wrist-worn sensors.

Table I Walking Tests

\begin{tabular}{|l|l|l|}
\hline $\begin{array}{l}\text { Experimental } \\
\text { Condition }\end{array}$ & $\begin{array}{l}\text { Type of Gait } \\
\text { Aid }\end{array}$ & Gait-aid Location \\
\hline 1 & None & NA \\
\hline 2 & Cane & $\begin{array}{l}\text { Dominant hand (same side as } \\
\text { sensor) }\end{array}$ \\
\hline 3 & Cane & $\begin{array}{l}\text { Nondominant hand (opposite side } \\
\text { as sensor) }\end{array}$ \\
\hline 4 & Single crutch & $\begin{array}{l}\text { Dominant hand (same side as } \\
\text { sensor) }\end{array}$ \\
\hline 5 & Single crutch & $\begin{array}{l}\text { Nondominant hand (opposite side } \\
\text { as sensor) }\end{array}$ \\
\hline 6 & $\begin{array}{l}\text { Two crutches } \\
\text { walker }\end{array}$ & NA \\
\hline 7 & walker & NA \\
\hline
\end{tabular}


Bland-Altman analyses were performed for all eight walking tests to compare agreement between measurement techniques (wrist counter vs manual step count and hip counter vs manual step count). The $x$-axes in the figures represents the average step count between the two measurement techniques for each participant $(\mathrm{A}+\mathrm{B} / 2)$, while the $y$-axes represents the difference in step count between the two measurement techniques $(\mathrm{A}-\mathrm{B})$. Solid horizontal lines represent the bias, which is the mean of step-count differences between the two measurement techniques across all participants for that test. Dashed horizontal lines represent $95 \%$ limits of agreement (LOAs), which show the range of values within $95 \%$ CIs: one can predict difference values between the measurement techniques will fall. LOA is calculated as $b \pm 1.96 \mathrm{~S}_{b}$, where $\mathrm{b}$ is the bias and $\mathrm{S}_{\mathrm{b}}$ the $\mathrm{SD}$ of the bias.

\section{Results}

The eight walking tests and mean step counts for each group are shown in Table 2. For nine subjects with no gait aids, the mean step count was significantly different between the hip group $(104.2 \pm 4.6)$ and the actual measured count $(101.3 \pm 3.3 ; p=0.04)$. For the stationary walker, the wrist counter $(65.8 \pm 44.8)$ and hip counter (36.1 \pm 51.6$)$ showed a significant difference $(p=0.01)$. In the four-wheeled walker test, the wrist group had an extremely low mean count of $8.7 \pm 13.9$, which was significantly different from the actual count of $100.0 \pm 0(p<0.0001)$.

Our findings showed a large variability in step-count values when measured with either Fitbit device. Figure 1 shows mean step counts of both Fitbits compared to the actual step count of the given walking test. Large SDs of the mean are seen throughout all Fitbit measured counts. However, the hip counter consistently recorded values closer to the actual step count when compared to the wrist counter. This held true for six of the eight walking tests: no gait aid (hip $=104.2$; actual $=101.3$; wrist $=$ 94.7), cane nondominant side (hip $=92.7$; actual $=100.4$; wrist $=83.9)$, crutch dominant side (hip $=96.1$; actual $=$ 100.1; wrist $=83.7)$, crutch - nondominant side (hip $=$ 97.2; actual $=100.1$; wrist $=107.3)$, crutch - both sides (hip $=90.3$; actual $=100.2$; wrist $=66.7$ ), and the fourwheeled walker $($ hip $=73.6$; actual $=100$; wrist $=8.7$ ). The wrist counter was more accurate when using a cane with the Fitbit on the dominant side (wrist $=88.8$; actual $=$ 100.9; hip $=72.8$ ) and while using a stationary walker $($ wrist $=65.8$; actual $=100.2 ;$ hip $=36.1)$.

Agreement between measurement techniques (wrist counter versus manual step count and hip counter versus manual step count) was assessed using Bland-Altman plots (Figure 2). Mean overall agreement for subjects walking without gait aids in the hip group was excellent, showing a bias of -2.9 and LOAs between -8.72 and 2.95 . With gait aids, Fitbits worn at both the wrist and the hip showed minimal agreement, with wide LOAs throughout all walking tests. For canes or crutches, the Bland-Altman plots had a range of bias values between both the hip and wrist counters of -7.22 to 33.56 , with LOAs from -98.55 to 124.2. The wrist counter during the four-wheeled walking test showed extremely little agreement with the actual step count, with bias of 91.33 and LOAs 64.1-118.6, illustrating exceptionally unreliable step counts.

\section{Discussion}

This study aimed to assess the use of Fitbits as a tool for measuring the physical activity of individuals using gait aids. Because walking aids are used by many people with chronic impairments, having a reliable method of measuring activity in this population can help guide rehabilitation and serve as a research tool.

Table 2 Summary of Walking Tests: Means \pm SD of step Counts

\begin{tabular}{|l|l|l|l|l|}
\hline Walking Test & Wrist & Actual & Hip & p-values \\
\hline No gait aids & $94.7 \pm 13.8$ & $101.3 \pm 3.3^{*}$ & $104.2 \pm 4.6^{*}$ & $0.04^{*}$ \\
Cane, dominant side & $88.8 \pm 30.9$ & $100.9 \pm 2.3$ & $72.8 \pm 42.7$ & $\mathrm{NS}$ \\
Cane, nondominant side & $83.9 \pm 33.4$ & $100.4 \pm 0.7$ & $92.7 \pm 35.0$ & $\mathrm{NS}$ \\
Crutch, dominant side & $83.7 \pm 29.8$ & $100.1 \pm 0.3$ & $96.1 \pm 38.1$ & $\mathrm{NS}$ \\
Crutch, nondominant side & $107.3 \pm 26.5$ & $100.1 \pm 0.3$ & $97.2 \pm 35.7$ & $\mathrm{NS}$ \\
Crutches, both sides & $66.7 \pm 46.2$ & $100.2 \pm 0.4$ & $90.3 \pm 55.3$ & $\mathrm{NS}$ \\
Stationary walker & $65.8 \pm 44.8$ & $100.2 \pm 0.4^{*}$ & $36.1 \pm 51.6^{*}$ & $0.0 I^{*}$ \\
Four-wheeled walker & $8.7 \pm 13.9 *$ & $100.0 \pm 0^{*}$ & $73.6 \pm 53.0$ & $<0.000 I^{*}$ \\
\hline
\end{tabular}

Note: $* p<0.05$.

Abbreviation: NS, not significant. 


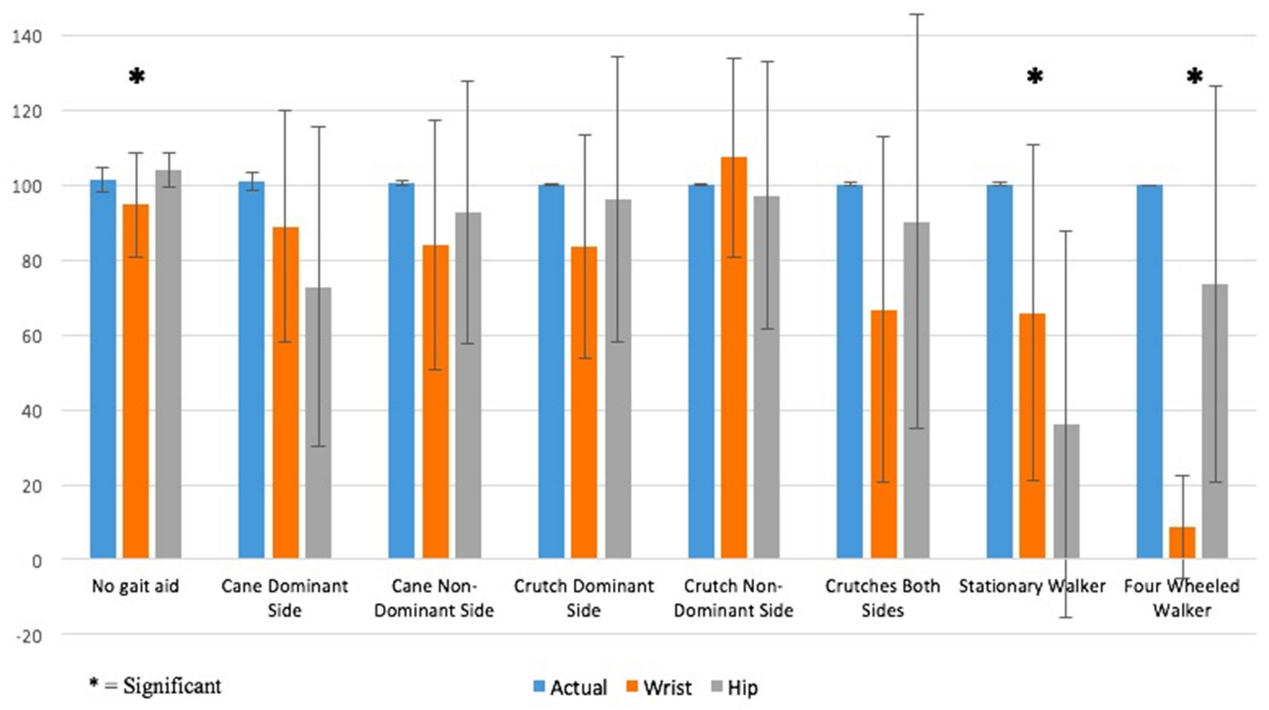

Figure I Means \pm SD of step counts for each walking test (*significant).

Our findings showed that in healthy volunteers using gait aids, the Fitbit regularly underestimated step count. This pattern held true across all walking tests, indicating little agreement and wide limits when compared to the actual step count, as shown by the Bland-Altman plots. When comparing the two groups, the plots showed that hip counters were stronger measures of actual count values. The following testing scenarios demonstrated Fitbit agreement of within $10 \%$ of actual step values: hip counter with cane - nondominant side ( $b=7.8$; $\mathrm{LOA}=-60.7$ to 76.3$)$, hip counter with single crutch on both dominant $(b=4$; $\mathrm{LOA}=-70.5$ to 78.5$)$ and nondominant sides $(\mathrm{b}=2.9$; $\mathrm{LOA}=66.9$ to 72.68$)$, and double crutches $(\mathrm{b}=9.9$; LOA $=98.6-118.3$ ), and wrist counter with single crutch on nondominant side $(b=-7.2 ; \mathrm{LOA}=59.2-44.8)$. In any of these given scenarios, the counter showed step agreement of within $10 \%$ of the actual step value. These findings suggest that Fitbits may be useful in approximating physical activity levels with gait aids in certain scenarios and may still be used in the rehabilitation setting.

One possible explanation for the vast differences and inconsistent agreement may be due to walking speeds with gait aids that do not closely mimic those of a healthy individual. Fitbits use an acceleration-threshold algorithm and accelerometers to quantify steps. ${ }^{10}$ The use of gait aids alters gait patterns, reducing the acceleration detected. This has also been shown in other studies. For example, an assessment of Fitbit technology in cardiac patients also found that there was inaccurate assessment of activity. ${ }^{11}$ Thorup et al demonstrated that slow-moving cardiac disease patients performing treadmill-walking tests at speeds $<3.6 \mathrm{~km} / \mathrm{h}$ caused inaccuracies in step counts with the Fitbit. It is possible that steps taken by participants while walking with gait aids were not fast enough to meet the Fitbit acceleration threshold, resulting in the consistently underestimated values.

The irregularity and inconsistency of participants' gait patterns while using a walking aid may also have influenced the measurement count. For example, when walking with a stationary walker, there is more wrist versus hip movement than for patients walking without a gait aid. This illustrates the poor agreement of the hip group in the stationary walker test, which had bias of 64.1 \pm 51.7 and LOAs of -37.3 to 165.5 . When walking with a four-wheeled walker, there is no wrist movement whatsoever. This led to an extremely underestimated step count, resulting in bias of $91.3 \pm 13.9$ and LOAs of 64.1118.6. These findings demonstrate how gait patterns have a vast impact on the sensitivity and detectability of steps on Fitbit devices. Furthermore, this is consistent with previous validation studies that have shown that for patients who have chronic illnesses and impaired gait patterns,step counts were underestimated. ${ }^{10,12-14}$

It was found that the hip was the most accurate location in detecting counts. In six of eight walking tests, the hip counter showed lower mean differences from the actual step count when compared to the wrist counter. This is consistent with previous results showing the hip to be the most accurate wearing location. ${ }^{15}$ The measurements in most agreement with the actual count were with the hip 

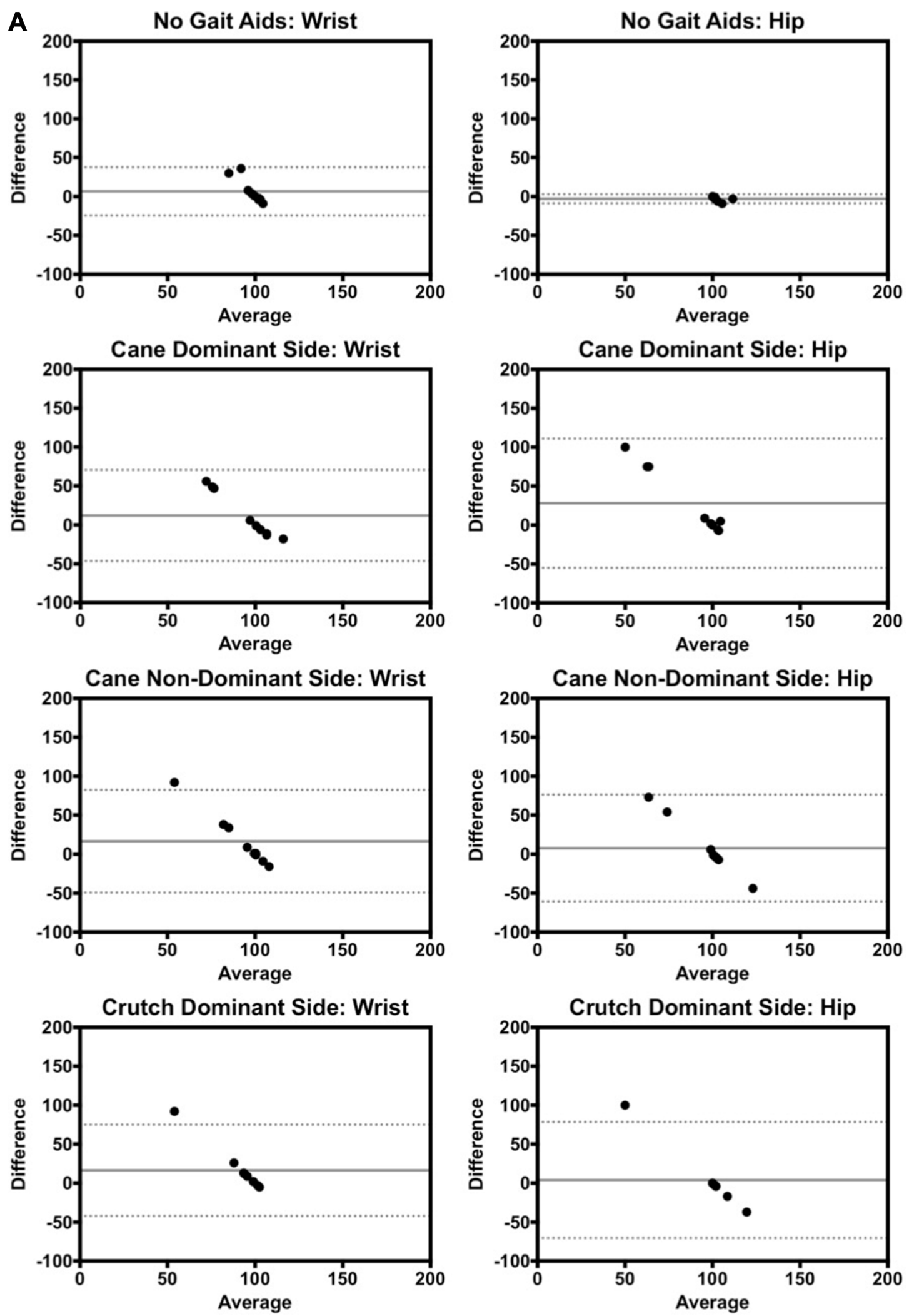

Figure 2 Continued. 

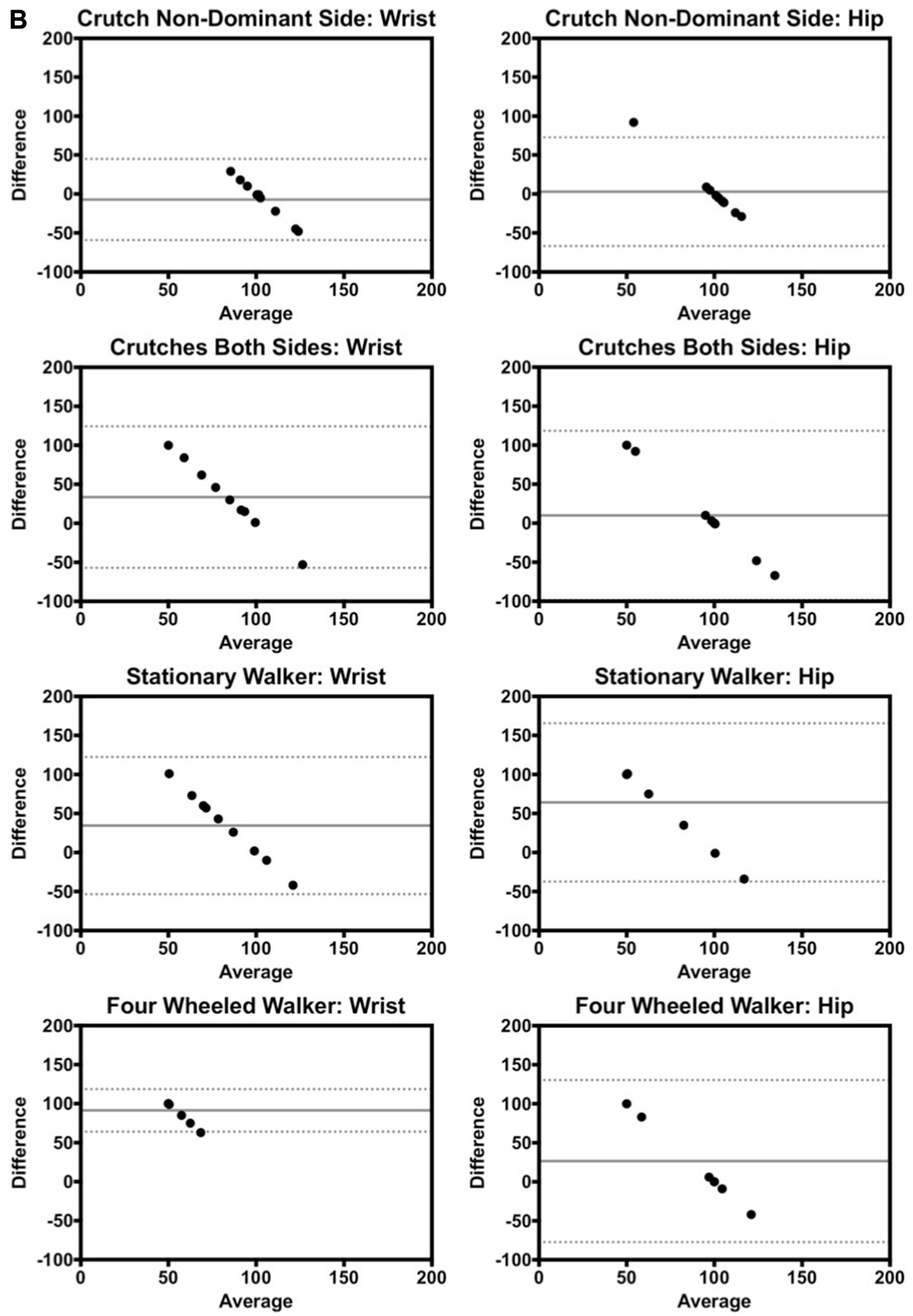

Figure 2 (A) Bland-Altman plots for no gait aids, canes, or crutches for each walking test, measured in number of steps. (B) Bland-Altman plots for crutches and walkers for each walking test, measured in number of steps. 
counter worn on the nondominant side during the singlecrutch walking test (absolute mean difference of 2.89 \pm 35.6 ).

There were several limitations in this experiment. The use of healthy volunteers without any gait disturbance may overestimate the accuracy of the trackers, given the minimum thresholds required to measure a step, as they may move faster than individuals with physical impairments. The measure of gait speed of these individuals may have helped explain the inaccurate step counts and may represent a methodological limitation of this study. Furthermore, the facility used required participants to walk in a straight, relatively long track and turn at the end of the $20 \mathrm{~m}$ hallway. This may not reflect daily-life context and may be less challenging than obstacle avoidance or shorter walking periods, where errors may be expected to increase. Participants were only asked to conduct each walking test once, so repeating the experiment would increase the reliability of our results.

Future considerations may include assessing the accuracy of the Fitbit at different anatomical locations. Singh et al demonstrated that at lower speeds, the Fitbit was more accurate when distally located on the lower limb, such as the ankle. ${ }^{16}$ Since regular sway of the forearm is altered or inhibited by the use of gait aids, it is reasonable to assume that the accuracy of the wrist counter would be low. Future studies may include the application of wearable step counters to more distal body parts where accuracy is shown to be higher.

In conclusion, we performed walking tests to validate the Fitbit's step-counter capabilities with gait aids. We found that agreement was within $10 \%$ of actual step values for hip counters using a cane on the nondominant side, a single crutch on the dominant or nondominant side, double crutches, and wrist counters using a single crutch on the nondominant side. Agreement was $>10 \%$ for both hip and wrist counters with a cane on the dominant side and substantially greater $(>25 \%)$ in both walker groups, thus indicating that step counters may not accurately interpret physical activity in certain situations. If using step counters to measure activity in patients using gait aids, we suggest using a hip counter on the nondominant side, but understand that if using a step counter with a walker, it is less accurate. The largest discrepancy was seen in walkers, as the change in gait patterns significantly underestimated step values. This study suggests that these widely commercially available step counters have poor reliability with gait aids, especially walkers, which should be taken into account for the research and clinical setting.

\section{Ethics Approval and Informed Consent}

Ethics were approved by the London Health Sciences Centre institutional ethics review board. This study was conducted in accordance with the Declaration of Helsinki. Informed consent to participate in the study was obtained from all subjects prior to commencement of this study. All study participants gave consent to have their data published.

\section{Disclosure}

Dr James L Howard reports grants, personal fees, and institutional support from Depuy, Stryker, Smith and Nephew, and Intellijoint outside the submitted work. Dr Brent A Lanting reports consultancy fees, principal investigator grant support, and institutional support from Stryker, DePuy, and Smith and Nephew, as well as institutional support from Zimmer. Dr Edward M Vasarhelyi reports grants and personal fees for institutional and research support from DePuy, Zimmer Biomet, Hip Innovation Technology, Stryker, and Smith and Nephew outside the submitted work. The authors report no other conflicts of interest in this work.

\section{References}

1. Kooiman TJ, Dontje ML, Sprenger SR, Krijnen WP, van der Schans CP, de Groot M. Reliability and validity of ten consumer activity trackers. BMC Sports Sci Med Rehabil. 2015;7:24. doi:10.1186/s13102-015-0018-5

2. Harding P, Holland AE, Delany C, Hinman RS. Do activity levels increase after total hip and knee arthroplasty? Clin Orthop Relat Res. 2014;472:1502-1511. doi:10.1007/s11999-013-3427-3

3. Hootman JM, Macera CA, Ham SA, Helmick CG, Sniezek JE. Physical activity levels among the general US adult population and in adults with and without arthritis. Arthritis Rheum. 2003;49:129-135. doi:10.1002/art.10911

4. Westerterp KR. Assessment of physical activity: a critical appraisal. Eur J Appl Physiol. 2009;105:823-828. doi:10.1007/s00421-0091000-2

5. Sallis JF, Saelens BE. Assessment of physical activity by self-report: status, limitations, and future directions. Res Q Exerc Sport. 2000;71: S1-4. doi:10.1080/02701367.2000.11082780

6. Fulk GD, Combs SA, Danks KA, Nirider CD, Raja B, Reisman DS. Accuracy of 2 activity monitors in detecting steps in people with stroke and traumatic brain injury. Phys Ther. 2013;94:222-229. doi:10.2522/ptj.20120525

7. Schaffer SD, Holzapfel SD, Fulk G, Bosch PR. Step counter accuracy and reliability of two activity tracking devices in people after stroke. Physiother Theory Pract. 2017;33(10):788-796. doi:10.1080/ 09593985.2017.1354412

8. Macko RF, Haeuber E, Shaughnessy M, et al. Microprocessor-based ambulatory activity monitoring in stroke patients. Med Sci Sports Exerc. 2002;34:394-399. doi:10.1097/00005768-200203000-00002

9. Mudge S, Stott NS, Walt SE. Criterion validity of the stepwatch activity monitor as a measure of walking activity in patients after stroke. Arch Phys Med Rehabil. 2007;88:1710-1715. doi:10.1016/j. apmr.2007.07.039 
10. Cyarto EV, Myers AM, Tudor-Locke C. Pedometer accuracy in nursing home and community-dwelling older adults. Med Sci Sports Exerc. 2004;36:205-209. doi:10.1249/01.MSS.0000113476.62469.98

11. Thorup C, Andreasen J, Sørensen E, Grønkjær M, Dinesen B, Hansen J. Accuracy of a step counter during treadmill and daily life walking by healthy adults and patients with cardiac disease. $B M J$ Open. 2017;7(3):e011742. doi:10.1136/bmjopen-2016-011742

12. Pitta F, Troosters T, Spruit MA, Decramer M, Gosselink R. Activity monitoring for assessment of physical activities in daily life in patients with chronic obstructive pulmonary disease. Arch Phys Med Rehabil. 2005;86:1979-1985. doi:10.1016/j.apmr.2005.04.016

13. Langer D, Gosselink R, Sena R, Burtin C, Decramer M, Troosters T. Validation of two activity monitors in patients with COPD. Thorax. 2009;64:641-642. doi:10.1136/thx.2008.112102
14. Larkin L, Nordgren B, Purtill H, Brand C, Fraser A, Kennedy N. Criterion validity of the activPAL activity monitor for sedentary and physical activity patterns in people who have rheumatoid arthritis. Phys Ther. 2015. doi:10.2522/ptj.20150281

15. Chow J, Thom J, Wewege M, Ward R, Parmenter B. Accuracy of step count measured by physical activity monitors: the effect of gait speed and anatomical placement site. Gait Posture. 2017;57:199-203. doi:10.1016/j.gaitpost.2017.06.012

16. Singh AK, Farmer C, Van Den Berg ML, Killington M, Barr CJ. Accuracy of the FitBit at walking speeds and cadences relevant to clinical rehabilitation populations. Disabil Health J. 2016;9:320-323. doi:10.1016/j.dhjo.2015.10.011

\section{Publish your work in this journal}

Orthopedic Research and Reviews is an international, peer-reviewed, open access journal that focusing on the patho-physiology of the musculoskeletal system, trauma, surgery and other corrective interventions to restore mobility and function. Advances in new technologies, materials, techniques and pharmacological agents are particularly welcome. The manuscript management system is completely online and includes a very quick and fair peer-review system, which is all easy to use. Visit http://www.dovepress.com/testimonials.php to read real quotes from published authors. 